Research Journal of Biological Sciences 5 (9): 632-637, 2010

ISSN: $1815-8846$

(C) Medwell Journals, 2010

\title{
The Role of Dates (Phoenix dactylifera) Aqueous Extract in Improving the Plasma Lipid Profiles of Diet-Induced Hypercholesterolemic Rabbits
}

\author{
${ }^{1}$ Nawal S. Hasan, ${ }^{2}$ Zulkhairi H. Amom, ${ }^{2}$ A.I. Nor, ${ }^{2}$ Daryl J. Arapoc and ${ }^{3}$ Azrina Azlan \\ ${ }^{1}$ Department of Physiology, Faculty of Medicine and Health Sciences, \\ Seventh of April University, 110 Jamal Abdonasser Street, Zawia, Libya \\ ${ }^{2}$ Department of Human Anatomy, Division of Physiology, \\ ${ }^{3}$ Department of Nutrition and Dietetics, Faculty of Medicine and Health Sciences, \\ University Putra Malaysia, 43400 Serdang, Selangor, Malaysia
}

\begin{abstract}
The influence of dates extract on lipid profile and atherosclerotic lesion formation in hypercholesterolemic induced rabbit were investigated. About 49 male New Zealand White (NZW) rabbits were divided into 7 groups. The Normal Control (NC) group, Hypercholesterolemic Control ( $\mathrm{HC}$ ) group was given $0.5 \%$ cholesterol diet, Simvastatin Control (SC) group was given $0.5 \%$ cholesterol diet $+5 \mathrm{mg} \mathrm{kg}^{-1}$ simvastatin. Treatment groups $\mathrm{T} 125, \mathrm{~T} 250, \mathrm{~T} 500$ and $\mathrm{T} 1000$ were given $0.5 \%$ cholesterol diet with supplementation of 125 , 250,500 and $1000 \mathrm{mg} \mathrm{kg}^{-1}$ of dates extract, respectively for 10 weeks. Blood was collected from ear vein for plasma analysis and the whole aortas were excised for microscopy study. The supplementation of 125 and $250 \mathrm{mg} \mathrm{kg}^{-1}$ of date extract reduced plasma Total Cholesterol (TC), Low-Density Lipoprotein (LDL) and Triglycerides (TG) levels concomitantly groups supplemented with date extract (T125, T250, T500 and T1000) were significantly higher $(\mathrm{p}<0.05)$ in High Density Lipoprotein $(\mathrm{HDL})$ in diet induced hypercholesterolemic rabbit. The Atherogenic Index (AI) and sdLDL values were found to be lower in date extract treated groups compared to $\mathrm{HC}(\mathrm{p}<0.05)$. The plasma total antioxidant activity in groups, treated with date extract $(\mathrm{T} 125, \mathrm{~T} 250$, $\mathrm{T} 500$ and $\mathrm{T} 1000)$ were significantly higher $(\mathrm{p}<0.05)$ in compared to HC group at 10 th week. No foam cell formation was visible in the aorta of rabbits in $\mathrm{NC}$ and T250 groups. However, there was visible foam cell formation in the aorta of groups $\mathrm{HC}, \mathrm{SC}, \mathrm{T} 125, \mathrm{~T} 500$ and $\mathrm{T} 1000$. Results showed that plasma lipid concentration was significantly reduced at the end of experiment in groups supplemented with date extract.
\end{abstract}

Key words: Dates, Phoenix dactylifera L., hypercholesterolemia, atherosclerosis, antioxidant, influence

\section{INTRODUCTION}

Cardiovascular Disease (CVD) is a leading cause of death. An estimated 17 million people die every year with 7.6 million deaths accounted for by coronary heart disease (Najafipour et al., 2010). Moreover, atherosclerosis is one of the major contributors to pathogenesis of coronary and cerebrovascular disease. Dietary cholesterol promotes the development of coronary plaque formation which increases the risk of ischemic heart disease (Schwartz et al., 2001). Hypercholesterolemic atherosclerosis is characterized by having a close relationship with inflammatory and oxidative stress processes reflective of the harmful effects of reactive free radicals within the arterial wall (Real et al., 2010). Strong evidence suggests that oxidative stress is one of the causative factors implicated in the progression of atherosclerosis in part by lipid oxidation and altered endothelial function (Real et al., 2010). In the presence of oxidative stress, oxidation of LDL occurs at an early stage of the process. Oxidized LDL (oxLDL) is highly reactive and cytotoxic to a variety of vascular cells (Chisolm and Steinberg, 2000). Date fruits are rich in phytochemicals such as phenolics, flavonoids, sterols, carotenoids, procyanidins and anthocyanins. The amounts of these compounds contribute to the nutritional and organoleptic characteristics of the fruits (Hasan et al., 2010; Baliga et al., 2010). Dates are considered an important ingredient of various atonic and aphrodisiac confections (Khare, 2007). Dates are also traditionally used to treat hypertension and diabetes (Tahraoui et al., 2007).

In addition, dates were reported to be protective against ulcer (Al-Qarawi et al., 2005), anti-tussive, expectorant, demulcent, laxative, diuretic and restorative (Khare, 2007). Despite of several health promoting effects of dates has been documented, the effect on cholesterol

Corresponding Author: Nawal S. Hasan, Department of Physiology, Faculty of Medicine and Health Sciences, Seventh of April University, 110 Jamal Abdonasser Street, Zawia, Libya 
metabolism and hypercholesterolemia is still scant hence, require call for detailed report. This study was designed to investigate the lipid-lowering activities of dates from Libya. Scientific clarifications drawn from this study will provide information on dates as an alternative source of natural antioxidants that can contribute to better health and well-being.

\section{MATERIALS AND METHODS}

Preparation of extract: Fresh matured dates of the Bekraray variety native to Libya were purchased from Libyan market and were selected in such a way as to be identical in terms of the mass ( $7-10 \mathrm{~g}$ ), colour (light brown) and ripening stage. The pericarps were separated from the seeds and minced. A concentration of $10 \%$ aqueous pulp extract was prepared by soaking $100 \mathrm{~g}$ of the fresh pulp (equivalent to 10 date pods) in sufficient volume of distilled water to produce a $1 \mathrm{~L}$ solution and mixed thoroughly. The mixture was incubated in a shaking water bath at $60^{\circ} \mathrm{C}$ for $6 \mathrm{~h}$ and subsequently filtered. Once filtered, the filtrates were freeze-dried and kept at $-80^{\circ} \mathrm{C}$ until use.

Animals and experimental design: About 49 male $\mathrm{New}$ Zealand White (NZW) rabbits with an average body weights falling within the range of $2.2-2.8 \mathrm{~kg}$ were used throughout this experiment. The rabbits were placed in individual stainless steel cages and acclimatized for 1 week with intervals of equal light-dark exposure and free access to drinking tap water and normal rabbit chow. Following acclimatization, the animals were randomly segregated into 7 groups of seven rabbits each to be later subjected to the different experimental treatments corresponding to various food and/or drug or combinations of both. The food and drug treatment combinations were normal standard rabbit chow (normal control) a $0.5 \%$ cholesterol diet (hypercholesterolemic control) a $0.5 \%$ cholesterol diet $+5 \mathrm{mg} \mathrm{kg}^{-1}$ statin ( simvastatin control) a $0.5 \%$ cholesterol diet $+125 \mathrm{mg} \mathrm{kg}^{-1}$ of dates aqueous extract a $0.5 \%$ cholesterol diet+250 mg kg-1 of dates aqueous extract a $0.5 \%$ cholesterol diet $+500 \mathrm{mg} \mathrm{kg}^{-1}$ of dates aqueous extract and lastly a $0.5 \%$ cholesterol diet $+1000 \mathrm{mg} \mathrm{kg}^{-1}$ of dates aqueous extract. The study was designed for 10 weeks.

The rabbits were sacrificed by exsanguinations. A midline thoracotomy was performed and the aorta was excised for histomorphometeric analysis. This experiment was approved by the Animal Care and Use Committee (ACUC), Faculty of Medicine and Health Sciences, University Putra Malaysia (UPM), Malaysia.
Lipid profile measurement: Blood samples were collected at week 0 and 10th. Analysis of lipid profiles included measuring plasma Total Cholesterol (TC), triglycerides (TG), High Density Lipoprotein (HDL) and Low Density Lipoprotein (LDL) levels using a Roche kit (Penzberg, Germany). All parameters were measured spectrophotometrically with Hitachi chemistry analyser. The tests utilized the principle of enzymatic colorimetric assay to read the sample.

Antioxidant activity: The plasma Total Antioxidant Activity (TAA), Glutathione Peroxidase (GSH-Px) and superoxide dismutase levels were determined to assess the antioxidant activity. Total antioxidant status was measured by monitoring radical cation formation from 2,2-azino-di-(3-ethylbenzthioazoline-6-sulfonate) (ABTS) incubated with a peroxidase (metmyoglobin) and $\mathrm{H}_{2} \mathrm{O}_{2}$ to produce a radical cation with a stable blue colour which was measured at $600 \mathrm{~nm}$. The Colorimetric method was programmed into a Cobas Mira autoanalyser, using a Randox kit (Country Antrim, UK). Glutathion peroxidase (GSH-Px) is an important antioxidant enzyme involved in the catalyses the reduction of $\mathrm{H}_{2} \mathrm{O}_{2}$ to water. The role of $\mathrm{SOD}$ is to accelerate the dismutation of the toxic superoxide radical $\left(\mathrm{O}_{2}\right)$, produced during oxidative energy processes to hydrogen peroxide and molecular oxygen. This method employs xanthine and Xanthine Oxidase (XOD) to generate superoxide radicals which react with 2 (4-iodophenyl)-3-(4-nitrophenol)-5-phenyltetrazolium chloride (I.N.T) to form a red formazan dye.

The superoxide dismutase activity is then measured by the degree of inhibition of this reaction. GSH-Px and SOD were measured with a Cobas Mira autoanalyser using a Roche kit (Penzberg, Germany).

Evaluation of atherosclerosis lesions: For histological analysis, paraffin-embedded tissue sections of aortic arch were stained with Hematoxylin and $\operatorname{Eosin}(\mathrm{H}$ and $\mathrm{E})$ stain. The thickness of foam cells and fatty streak were measured with a light microscope equipped with an image analyser system (Olympus, Germany).

Statistical analysis: Analysis of Variance (ANOVA) and Tukey HSD were performed to compare the mean between groups. Significance was accepted at $\mathrm{p}<0.05$.

\section{RESULTS}

Lipid profiles: The concentrations of TC, HDL, LDL and TG at week 0 and 10 are shown in Table 1 . The TC levels in $\mathrm{HC}$ group were significantly increased $\left(19.59 \pm 0.52 \mathrm{mmol} \mathrm{L}^{-1}\right)$ following high cholesterol diet 
Res. J. Biol. Sci., 5 (9): 632-637, 2010

\begin{tabular}{|c|c|c|c|c|c|c|c|c|c|}
\hline Groups & $\begin{array}{l}\mathrm{TC} \\
-\cdots\end{array}$ & $\mathrm{HDL}$ & LDL & $\mathrm{TG}$ & $\begin{array}{l}\text { LDL:HDL } \\
-\left(\mathrm{mmol} \mathrm{L}^{-1}\right.\end{array}$ & TG:HDL & TAA & GSH-Px & SOD \\
\hline $\mathrm{NC}$ & $1.03 \pm 0.08^{*}$ & $0.56 \pm 0.06^{*}$ & $0.18 \pm 0.01^{*}$ & $0.38 \pm 0.07^{*}$ & $0.33 \pm 0.04 *$ & $0.65 \pm 0.093$ & $1.93 \pm 0.006$ & $765.41 \pm 74.37^{*}$ & $1.56 \pm 0.16^{*}$ \\
\hline $\mathrm{HC}$ & $19.59 \pm 0.56$ & $2.31 \pm 0.12$ & $9.39 \pm 0.94$ & $1.5 \pm 0.070$ & $4.42 \pm 0.36$ & $0.65 \pm 0.040$ & $1.91 \pm 0.000$ & $589.10 \pm 47.12$ & $2.86 \pm 0.39$ \\
\hline $\mathrm{SC}$ & $0.94 \pm 0.11 *$ & $4.75 \pm 0.66^{*}$ & $4.07 \pm 0.25 *$ & $0.25 \pm 0.03^{*}$ & $0.87 \pm 0.10^{*}$ & $0.05 \pm 0.008^{*}$ & $1.94 \pm 0.008$ & $786.48 \pm 80.78$ & $4.72 \pm 0.52^{*}$ \\
\hline T125 & $0.97 \pm 0.09^{*}$ & $9.04 \pm 0.37^{*}$ & $4.08 \pm 0.41 *$ & $0.53 \pm 0.07^{*}$ & $0.45 \pm 0.05^{*}$ & $0.06 \pm 0.008^{*}$ & $2.5 \pm 0.6400^{*}$ & $854.83 \pm 89.84$ & $4.88 \pm 0.60^{*}$ \\
\hline $\mathrm{T} 250$ & $7.05 \pm 0.57^{*}$ & $11.52 \pm 0.63^{*}$ & $3.72 \pm 0.55^{*}$ & $0.63 \pm 0.08^{*}$ & $0.32 \pm 0.05^{*}$ & $0.05 \pm 0.004^{*}$ & $2.53 \pm 0.640^{*}$ & $1278.07 \pm 162.0$ & $5.96 \pm 0.89^{*}$ \\
\hline T500 & $14.54 \pm 2.19^{*}$ & $12.09 \pm 0.41^{*}$ & $4.57 \pm 0.54 *$ & $0.66 \pm 0.15^{*}$ & $0.38 \pm 0.05^{*}$ & $0.05 \pm 0.005^{*}$ & $2.49 \pm 0.050^{*}$ & $800.64 \pm 50.83$ & $4.65 \pm 0.85^{*}$ \\
\hline $\mathrm{T} 1000$ & $16.99 \pm 1.22^{*}$ & $11.66 \pm 0.85^{*}$ & $6.12 \pm 0.16^{*}$ & $0.76 \pm 0.07^{*}$ & $0.53 \pm 0.04^{*}$ & $0.07 \pm 0.009^{*}$ & $2.43 \pm 0.050^{*}$ & $799.09 \pm 87.70^{*}$ & $4.50 \pm 0.64 *$ \\
\hline
\end{tabular}

Values are expressed as mean $\pm \mathrm{SD}(\mathrm{n}=7) ;{ }^{*} \mathrm{p}<0.05$ in comparison to hypercholesterolemic control group; $\mathrm{NC}=$ Negative Control; HC = Hypercholesterolemic Control; $\mathrm{SC}=0.5 \%$ cholesterol + simvastatin; $\mathrm{T} 125=0.5 \%$ cholesterol $+125 \mathrm{mg} \mathrm{kg}{ }^{-1}$ of date extract; $\mathrm{T} 250=0.5 \%$ cholesterol $+250 \mathrm{mg} \mathrm{kg}{ }^{-1}$ of date extract; $\mathrm{T} 500=0.5 \%$ cholesterol $+500 \mathrm{mg} \mathrm{kg}^{-1}$ of date extract; $\mathrm{T} 1000=0.5 \%$ cholesterol $+1000 \mathrm{mg} \mathrm{kg}^{-1}$ of date extract

consumption for 10 weeks when compare to those group fed with normal diet. Supplementation of date extract at

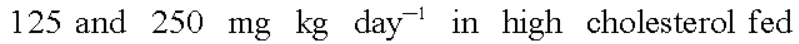
groups, lower the TC levels significantly in comparison with $\mathrm{HC}$ group $(\mathrm{p}<0.05)$ with an average of $0.97 \pm 0.09$ and $7.05 \pm 0.57 \mathrm{mmol} \mathrm{L}^{-1}$, respectively. The reductions of TC concentration correspond to a 95 and $64 \%$ in T125 and $\mathrm{T} 250$ groups, respectively compared to $\mathrm{HC}$ at 10 th week. Supplementation of dates extract at dose of 125 and $250 \mathrm{mg} \mathrm{kg} \mathrm{day}{ }^{-1}$ to rabbits fed with $0.5 \%$ cholesterol diet also reduced LDL levels significantly by 56 and $60 \%$ $(\mathrm{p}<0.05)$ than the HC group in 10th week whereas the level of TG was observed to be lowest $\left(0.532 \pm 0.074 \mathrm{mmol} \mathrm{L}^{-1}\right)$ on group treated with $125 \mathrm{mg} \mathrm{kg}^{-1}$ of date compared to $\mathrm{HC}$ group.

In contrary, the $\mathrm{HDL}$ level was significantly increased in all groups supplemented with date extract $(\mathrm{p}<0.05)$ compared with the $\mathrm{HC}$ group. The increased level of $\mathrm{HDL}$ in T125, T250, T500 and T1000 groups in 10th week were correspond by $74,79,80$ and $80 \%$, respectively, over the level characterised in the $\mathrm{HC}$ group. The $\mathrm{LDL}$ to $\mathrm{HDL}$ ratio and TG to $\mathrm{HDL}$ ratio at weeks 0 and 10 for all groups. A therosclerotic index (AI) which is the ratio of LDL to HDL level and sdLDL which is the ratio of TG to HDL level, significantly reduced in all groups supplemented with dates extract.

Antioxidant activity: The Total Antioxidant Activity (TAA) was found to be highest in T250 and correspond to $26 \%$ higher than the $\mathrm{HC}$ group in 10 weeks as shown in Table 1. The results showed that the GSH-Px activities was significantly decreased by $23 \%$ in animals fed with cholesterol-rich diet compared to the $\mathrm{NC}$ group at 10th week. However, the group supplemented with $250 \mathrm{mg} \mathrm{kg}$ day $^{-1}$ of date extract exhibited a significant increase in GSH-Px activity by $53 \%$ over that of the HC group (Table 1). In 10th week, group induced with high cholesterol diet resulted in a significant increase in the Superoxide Dismutase (SOD) activity by $45 \%$, over that of the control diet group $(\mathrm{p}<0.05)$. Interestingly, treatment of the hypercholesterolemia induced groups with date extract resulted in significant increment in the SOD activity than the HC group at 10th week by which the T250 group showed a remarkable increased activity of SOD (52\%) compare to that of the $\mathrm{HC}$ group in 10 weeks.

Thickness of foam cells: As shown in Fig. 1a-g, the aorta samples of the HC group exhibited a remarkable thickening of the intimal layer amounting to $633.09 \pm 52.2$ $\mu \mathrm{m}$. However, no foam cell formation in $\mathrm{NC}$ group or group fed with $250 \mathrm{mg} \mathrm{kg} \mathrm{day}{ }^{-1}$ of date aqueous extract was observed.

On the other hand, the rabbits treated with simvastatin and 125,500 and $1000 \mathrm{mg} / \mathrm{kg} /$ day of date aqueous extracts demonstrated a thickening of the intimal layers of the corresponding aortas. In addition, the thicknesses of the aortic intima in these groups were significantly different from one the other and correspondedto $242.39 \pm 10.73,318.89 \pm 37.99,180.71 \pm 28.29$ and $359.9 \pm 67.73 \mu \mathrm{m}$, respectively.

\section{DISCUSSION}

Risk of coronary heart disease seems to be positively associated with the plasma level of total cholesterol and LDL and inversely associated with its levels of $\mathrm{HDL}$ (Knopp et al., 2008; Kishida et al., 2002). This indicates that an increase intake of dietary cholesterol in animals led to hypercholesterolemia as evidenced by a significant increase in plasma total cholesterol concentration. In this experiment, the plasma LDL levels increased in groups fed with high cholesterol diet for 10 weeks, over those in the normal control group. These changes may be attributed to the excessive loads of cholesterol on the liver resulting to the down regulation of LDL receptors by the cholesterol and saturated fatty acids in the diet, thus causing in cholesterol being re-circulated in the blood (Mustad et al., 1997). Conversely, groups having high cholesterol diet concomitantly with dates extract supplementation, demonstrated a reduction of plasma TC and LDL levels significantly $(\mathrm{p}<0.05)$ compared with group without dates supplementation. 

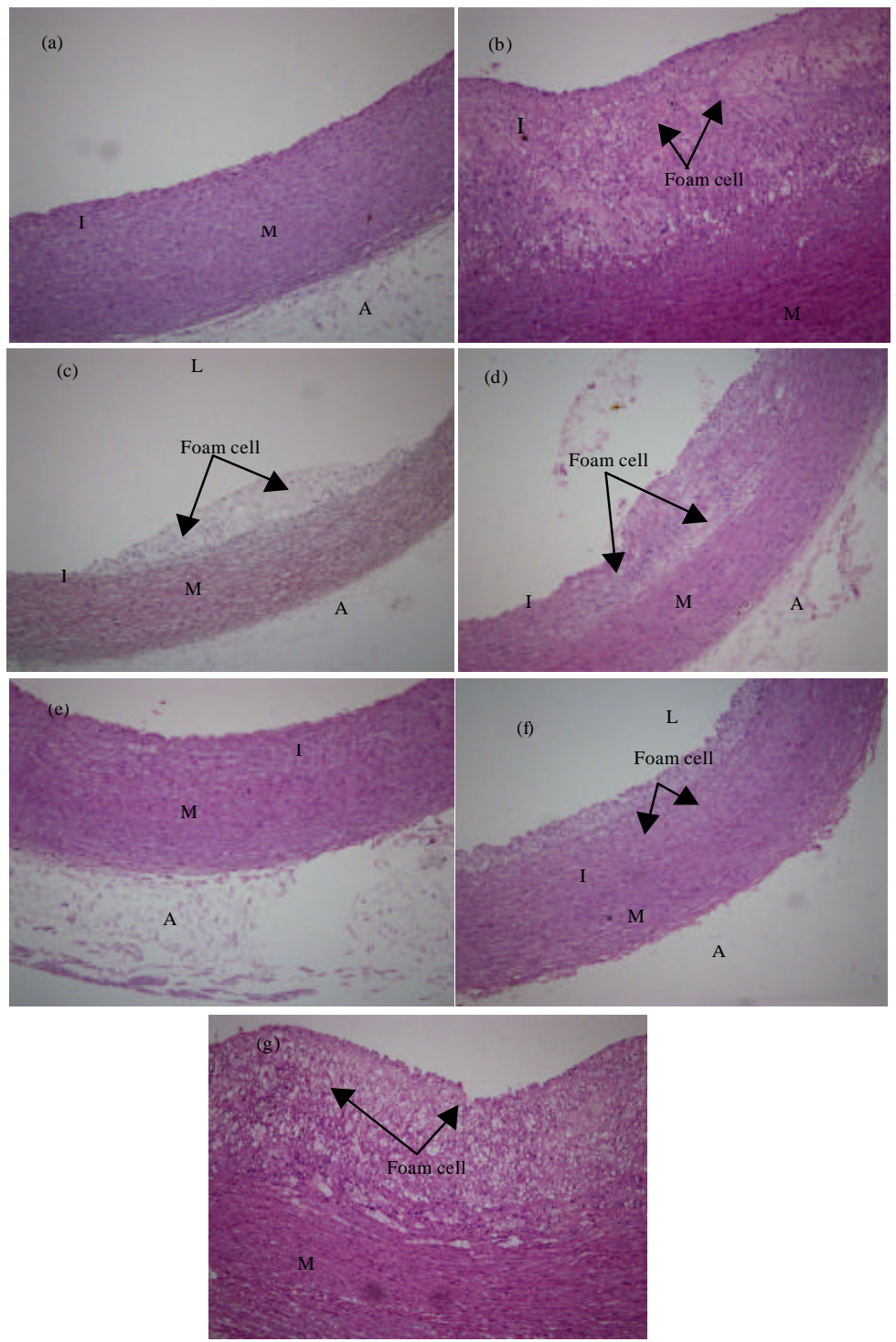

Fig. 1: Photomicrograph of aorta stained with $\mathrm{H}$ and $\mathrm{E}$ at LM x 10. $\mathrm{I}=$ Intima, $\mathrm{M}=$ Media, $\mathrm{A}=\mathrm{Adventitia}, \mathrm{L}=\mathrm{Lumen}$ group; a: NC; b: HC; c: SC; d: T125; e: T250; f: T500; g: T1000

This result bears resemblance with findings of Alsaif et al. (2007) who reported that dietary dates assist a significant reduction of plasma TC level during hypercholesterolemia. The relationship between plasma TC level and development of CHD has been well established (Castelli, 1984). However, association of CHD with increased levels of plasma Triglycerides (TG) is not clear (Austin, 1991). Some previous epidemiological studies reported that individuals with high plasma TG level are at high risk of $\mathrm{CHD}$ (Onat et al., 2006). In the present study, rabbits fed with high cholesterol diet for 10 weeks exhibited a significant elevation $(\mathrm{p}<0.05)$ in the TG level over the group, fed with normal rabbit diet.
However, the study revealed that the TG level has reduced by $65 \%$ upon date aqueous extract supplementations for 10 weeks compare with the $\mathrm{HC}$ group. On the other hand, the antiatherogenic lipoproteins such as $\mathrm{HDL}$, protect from atherosclerosis. It has been well documented on the pivotal role of HDL in the protection against CHD (Tsompanidi et al., 2010).

The role of $\mathrm{HDL}$ appears to facilitate the enhancement of reverse lipid transport pathway by translocation of stored cholesterol and other lipids from peripheral tissues including the arterial wall to the liver for further processing (Newton and Krause, 2002). This study demonstrated a favorable effect of dates in 
hypercholesterolemia and the results were in agreement with findings from previous studies (Maria et al., 2008; Zulkhairi et al., 2010). The present study also found that the total antioxidant activities in rabbits fed with high cholesterol diet were lower than rabbits fed with standard rabbit diet.

The reduction in the antioxidant activity could be due to the fact that hypercholesterolemia suppresses the antioxidant reserve and reduce the endogenous antioxidant enzymes in addition to the elevation in the concentrations of lipid peroxide products (Anila and Vijayalakshmi, 2002). On the other hand, supplemention of date aqueous extract during hypercholesterolemia, signifies the TAS activities compared to the animals fed with high cholesterol diet alone. The favorable effects of dates towards improvement on antioxidant enzymes activity was believes through the reinforcement of specific biosynthesis of antioxidant enzymes, mainly the $\mathrm{SOD}$ and GSH-Px in the liver. Considering the intrinsic stress-related markers (SOD and GSH-Px), these results suggest that date aqueous extract strongly enhance the efficiency of superoxide radicals dismutation to hydrogen peroxide (a much less harmful product) and increase the SOD activity following breakdown of hydrogen peroxide by GSH-Px. It should be highlighted that no data have so far been published on the effects of date extracts on antioxidant enzymes.

In agreement with a number of earlier related reports (Prasad, 1999; Lee and Prasad, 2003), results of this study showed that prolonged exposure to cholesterol diet triggers atherogenesis indicated with an increased wall thickness of the excised aorta. However, supplementation of dates aqueous extract demonstrated a reduction of atheroma plaque formation in the animal system. A causal relationship between lipid peroxidation and hypercholesterolemic atherosclerosis, suggesting the involvements of oxygen-derived free radicals (Green et al., 2001) in the initial stage of CVD and atherosclerosis. Increased concentrations of free radicals were associated with toxicological and pathological events in vivo including endothelial cell injury (Ohyashiki and Nunomura, 2000).

Alteration of endothelial cell function linked closely to the onset of the development of atherosclerosis (Ross, 1986). Accordingly, a diet rich with antioxidants can prevent the harmful effects of oxidative metabolism by quenching oxygen radicals, thus inhibiting LDL oxidative modification and delaying initiation of atherosclerosis (Singh et al., 2008). Diet from plant and fruit sources were rich in polyphenols which acted as an antioxidant. Therefore, one important therapeutic approach in procrastinate atherogenesis and CVD is minimizing the LDL oxidative modification. Dietary supplement such as dates palm could act as natural source of antioxidant and prevent free radical mediated diseases such as atherosclerosis.

\section{CONCLUSION}

The present study shows that date aqueous extract possesses a hypocholesterolemic and antioxidative effects hence, delaying the onset of atherosclerosis. The enhanced biosynthesis of antioxidant enzymes indicated with an increased SOD and GSH-Px activities apart from the phenolic concentration in dates could be the possible underlying mechanism of anti-atherosclerotic properties of dates palm. Further studies are required to identify, the probable role of dates leading to the hypocholesterolemic effects in vivo.

\section{ACKNOWLEDGEMENTS}

The researchers wishes to extend sincere gratitude to the government of Malaysia and University Putra Malaysia for granting the research and the Libyan government for providing a scholarship.

\section{REFERENCES}

Al-Qarawi, A.A., H. Abdel-Rahman, B.H. Ali, H.M. Mousa and S.A. El-Mougy, 2005. The ameliorative effect of dates (Phoenix dactylifera L.) on ethanol-induced gastric ulcer in rats. J. Ethnopharmacol., 98: 313-317.

Alsaif, M.A., L.K. Khan, A.A.H. Alhamdan, S.M. Alorf, S.H. Harfi, A.M. Al-Othman and Z. Arif, 2007. Effect of dates and gahwa (Arabian Coffee) supplementation on lipids in hypercholesterolemic hamsters. Int. J. Pharmacol., 3: 123-129.

Anila, L. and N.R. Vijayalakshmi, 2002. Flavonoids from Emblica officinalis and Mangifera indica-effectiveness for dyslipidemia. J. Ethnopharmacol., 79: 81-87.

Austin, M.A., 1991. Plasma triglyceride and coronary heart disease. Arterioscler. Thromb., 11: 2-14.

Baliga, M.S., B.R.V. Baliga, S.M. Kandathil, H.P. Bhat and P.K. Vayalil, 2010. A review of the chemistry and pharmacology of the date fruits (Phoenix dactylifera L.). Food Res. Int., 10.1016/j.foodres. 2010.07.004.

Castelli, W.P., 1984. Epidemiology of coronary heart disease: The Framingham study. Am. J. Med., 76: 4-12.

Chisolm, G.M. and D. Steinberg, 2000. The oxidative modification hypothesis of atherogenesis: An overview. Free Radical Biol. Med., 28: 1815-1826. 
Green, P., S. Glozman, L. Weiner and E. Yavin, 2001. Enhanced free radical scavenging and decreased lipid peroxidation in the rat fetal brain after treatment with ethyl docosahexaenoate. Biochim. Biophys. Acta, 1532: 203-212.

Hasan, N.S., Z.H. Amom, A.I. Nor, N. Mokhtarrudin, N.M. Esa and A. Azlan, 2010. Nutritional composition and in vitro evaluation of the antioxidant properties of various dates extracts (Phoenix dactylifera L.) from libya. Asian J. Clin. Nutr., 2: 208-214.

Khare, C.P., 2007. Indian Medicinal Plants: An Illustrated Review. Springer-Verlag, USA.

Kishida, T., H. Nogami, H. Ogawa and K. Ebihara, 2002. The hypocholesterolemic effect of high amylose cornstarch in rats is mediated by an enlarged bile acid pool and increased fecal bile acid excretion, not by cecal fermented products. J. Nutr., 132: 2519-2524.

Knopp, R.H., P. Paramsothy, B. Atkinson and A. Dowdy, 2008. Comprehensive lipid management versus aggressive low-density lipoprotein lowering to reduce cardiovascular risk. Am. J. Cardiol., 101: S48-S57.

Lee, P. and K. Prasad, 2003. Suppression of oxidative stress as a mechanism of reduction of hypercholesterolemic atherosclerosis by cyclooxygenase inhibitors. Int. J. Angiol., 12: 13-23.

Maria, A.M., M. Marta, E. Jeane, H. Rosaio, A. Amaya and L.F. Rosina, 2008. Effect of the long term intake of an egg white hydrolysate on the oxidative status and blood lipid profile of spontaneously hypertensive rats. Food Chem., 109: 361-367.

Mustad, V.A., T.D. Etherton, A.D. Cooper, A.M. Mastro, T.A. Pearson, S.S. Jonnalagadda and P.M. Kris-Etherton, 1997. Reducing saturated fat intake is associated with increased levels of LDL-receptors on mononuclear cells in healthy men and women. J. Lipid Res., 38: 459-468.

Najafipour, H., S. Joukar, R. Malekpour-Afshar, F. Mirzaeipour and H.R. Nasri, 2010. Passive opium smoking does not have beneficial effect on plasma lipids and cardiovascular indices in hypercholesterolemic rabbits with ischemic and non-ischemic hearts. J. Ethnopharmacol., 127: $257-263$.

Newton, R.S. and B.R. Krause, 2002. HDL therapy for the acute treatment of atherosclerosis. Atheroscler. Suppl., 3: 31-38.

Ohyashiki, T. and M. Nunomura, 2000. A marked stimulation of $\mathrm{Fe} 3+$-dependent lipid peroxidation in phospholipid liposomes under acidic conditions. Biochim. Biophys. Acta, 1484: 241-250.
Onat, A., I. Sariu, M. Yazici, G. Can, G. Hergenc and G.S. Avci, 2006. Plasma triglycerides, an independent predictor of cardiovascular disease in men: A prospective study based on a population with prevalent metabolic syndrome. Int. J. Cardiol., 108: 89-95.

Prasad, K., 1999. Action of serum cholesterol and hypercholesterolemia atherosclerosis in rabbits by secoisolariciresinol diglucoside isolated from flaxseed. Circulation, 99: 1355-1362.

Real, J.T., S. Martinez-Hervas, M.C. Tormosd, E. Domenech and F.V. Pallardoe et al., 2010. Increased oxidative stress levels and normal antioxidant enzyme activity in circulating mononuclear cells from patients of familial hypercholesterolemia. Metabol. Clin. Exp., 59: 293-298.

Ross, R., 1986. The pathogenesis of atherosclerosis: An update. N. Engl. J. Med., 314: 488-500.

Schwartz, G.G., A.G. Olsson and M.D. Ezekowitz, 2001. The mycoardial ischemia reduction with aggressive cholesterol lowering (MIR-ACL) study investigators. Effects of atorvastatin on early recurrent ischemic events in acute coronary syndromes. The MIRACL study: A randomized controlled trial. J. Am. Med. Assoc., 285: 1711-1718.

Singh, I., M. Mok, A.M. Christensen, A.H. Turner and J.A. Hawley, 2008. The effects of polyphenols in olive leaves on platelet function. Nutr. Metab. Cardiovasc. Dis., 18: 127-132.

Tahraoui, A., J. El-Hilaly, Z.H. Israili and B. Lyoussi, 2007. Ethnopharmacological survey of plants used in the traditional treatment of hypertension and diabetes in South-Eastern Morocco (Errachidia province). J. Ethnopharmacol., 110: 105-117.

Tsompanidi, E.M., M.S. Brinkmeier, E.H. Fotiadou, S.M. Giakoumi and K.E. Kypreos, 2010. HDL biogenesis and functions: Role of HDL quality and quantity in atherosclerosis. Atheroscler., 208: 3-9.

Zulkhairi, H.A., A.F. Khairunnuur, M.R.N. Hafipah, A. Azrina and M.A. Rasadah et al., 2010. An aqueous extract of Citrus mitis possesses antioxidative properties and improves plasma lipid profiles in rat induced with high cholesterol diet. J. Med. Plants Res., 4: 49-57. 